\title{
Integrated Monitoring and Supportive Visits as a Tool to Monitor New Products Transition and Quality of Care of Antiretroviral Treatment Programme in Nigeria
}

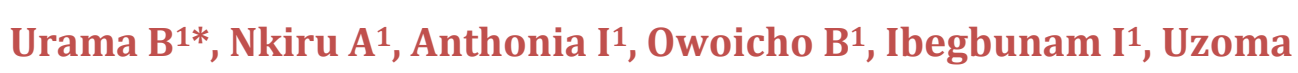 \\ $\mathrm{A}^{2}$, Chukwukaodinaka $\mathrm{N}^{2}$, Adelanwa $\mathrm{A}^{3}$ and Babatunji $\mathbf{O}^{3}$ \\ 1USAID Global Health Supply Chain-Procurement and Supply Management project, \\ Nigeria \\ ${ }^{2}$ National AIDS and STI Control Program, Nigeria \\ ${ }^{3}$ United State Agency for International Development, Nigeria
}

\section{Research Article \\ Volume 3 Issue 2}

Received Date: August 08, 2019

Published Date: September 17, 2019

DOI: $10.23880 /$ phoa-16000144

*Corresponding author: Bennett Urama, The USAID Global Health Supply Chain-Procurement and Supply Management (GHSC-PSM) project, Abuja, Nigeria, Tel: +2348037784392; Email: yarobee@gmail.com

\section{Abstract}

Background: Integrated monitoring and supportive visit (iMSV) to health facilities is a facilitative approach that promotes mentorship, team work, joint problem-solving, cross-learning, skill sharing and communication between programme staff and health care workers (HCWs) at the service delivery points. The National AIDS and STIs Control Programme (NASCP) instituted iMSV as part of the effort to monitor uptake of new antiretroviral drugs and quality of care of the antiretroviral treatment programme. A range of measures were taken to ensure that the iMSV team carry out the activities effectively to guide, support and assist health facility staff to become more competent in their work. This article highlights the processes of the iMSV and the outcome of the exercise.

Methodology: A standard assessment checklist was used. Data were collected from 154 health facilities through interviews, direct observation, document review and physical inventory. Health facilities were sampled using three criteria: sites with population of patients on antiretroviral therapy (ART) above $\geq 1000$; sites without logistics Management information report (LMIS) report in the last three cycles (July/August 2018, Sept/Oct 2018 and Nov/Dec 2018); sites with zero consumption of dolutegravir 50mg (DTG) and tenofovir/lamivudine/dolutegravir (TLD) from November/December 2018 LMIS data. Special consideration was given to states with highest HIV burden. Combined team from funders, implementing partners, national and state government took part in the exercise. Data entry and analysis were done using MS Excel. 
Results: Findings revealed that there are trained but inadequate HCWs of all cadres supporting the programme. It also showed that key elements of quality of care e.g. baseline laboratory investigations, WHO staging for treatment naïve patients, viral load testing, TB preventive therapy are been implemented but need to be improved. Necessary guidelines, standard operating procedures and job aids were available in most of the health facilities. The new antiretroviral drugs were generally available and most of the health facilities have commenced the use. There is slow pace of transitioning of existing adult and adolescent patients from previously preferred regimen to the new preferred regimen as approved by NASCP. Few incidents of HIV product stock out were recorded in some health facilities. Storage condition for the products is generally okay and laboratories carry out necessary quality control on reagents and kits batches before use.

Conclusion: iMSV is a promising approach to monitoring and improving uptake of new products and quality of care of the programme. Close monitoring and re-strengthening of the HCWs had a positive impact on the overall performance of the programme.

Keywords: Integrated Monitoring and Supportive Visit; Antiretroviral Treatment; Antiretroviral Drugs; Viral Load; Quality of Care; Health Care Workers

Abbreviations: TLD: Tenofovir Lamivudine Dolutegravir; TLE: Tenofovir Lamivudine Efavirenz; LNZ: Lamivudine Nevirapine Zidovudine; PSM: Procurement and Supply Management; M\&E: Monitoring \& Evaluation.

\section{Introduction}

In HIV/AIDS programme, ongoing monitoring visit is needed for health workers in the frontline of service delivery to perform to their full potential and deliver quality patient care. The monitoring visit plays an important role in the management of human resources to improve the quality of health care and health service delivery [1]. When it is supportive in nature, it can promote continuous improvements in the quality of care by providing the necessary leadership and support for quality improvement processes and by emphasizing mentorship, joint problem-solving, and two-way communication between programme staff and service providers $[2,3]$.

Ali, et al. [4] defined monitoring as the periodic collection and review of information on programme implementation, coverage and use for comparison with implementation plans. It identifies shortcomings before it is too late [4]. Marquez \& Kean defined supportive visit as a process that promotes quality at all levels of the health system by strengthening relationships within the system, focusing on the identification and resolution of problems, and helping to optimize the allocation of resources promoting high standards, teamwork, and better two-way communication [3]. This is different from the 'traditional' supervision, as it employs the concept of humanized support, as opposed to managerial control [5]. Unlike what happens in traditional supervision where supervisors often blame individuals rather than look for root causes in deficient processes [3]. The concept of integrating monitoring activities as part of supportive visit to the health facilities has been generally implemented by the National HIV Program. It is a process whereby programme staff guide and encourage service providers to optimize their performance in a supportive environment and recognize them when they attain a high level of performance [6]. The cornerstone of monitoring and supportive visit (MSV) is working with health staff to monitor performance, identify and correct problems, recognize good practices, help health workers to maintain their high-level of performance and proactively improve the quality of service [7].

In the past, different components of HIV/AIDS programme in Nigeria (Treatment, Laboratory, Procurement and Supply Management etc.) implemented separate monitoring and supportive visits (MSV) to the health facilities. In this system, programme staff typically employs checklists and assessment forms to facilitate MSV that are specific to their thematic areas. While this can identify, and address issues and challenges specific to different thematic areas, it limits the opportunity to better 
understand and identify all programmatic issues and challenges and to develop a holistic plan to address the issues. It was against this background and the recent introduction of new antiretroviral drugs and ongoing regimen transition from previously preferred adult and adolescent first line regimen tenofovir/lamivudine/efavirenz (TLE) and lamivudine/nevirapine/zidovudine (LNZ) to the new preferred regimen tenofovir/lamivudine/dolutegravir (TLD), that the National AIDS and STIs Control Programme (NASCP) instituted an integrated monitoring and supportive visit (iMSV) to health facilities implementing HIV/AIDS programme to assess implementation of national ART guidelines, regimen transition plan among others. In the Integrated approach adopted by NASCP- Treatment, Procurement and Supply Management (PSM), Laboratory and Monitoring \& Evaluation (M\&E) units of the health facilities were supported concurrently in one visit by multiple staff from different components of the programme and it focused on problem-solving to assure quality and meet client needs [3]. The iMSV approach which optimizes resources and improves efficiencies typically involved: performance observation and comparison of actual practices with standards; facilitative feedback on performance; provision of guidelines or technical updates; use of client input and data to ascertain opportunities for improvement; problem solving as a team; and follow-up of previously noted problems. The iMSV strengthens the relationship between the program staff and health care workers as it allows individuals to work together to assess facility performance and develop action plans to improve those results[3]. As a result, the leadership and skills of health workers at facilities is expected to increase, improving the quality of care delivered to patients. By strengthening the relationship between all workers and increasing their role and ownership of services provided, IMSV is intended to lead to improved health outcomes [8].

The National integrated MSV was led by the National AIDS and STIs Control Programme (NASCP) and National Agency for the Control of AIDS (NACA) with technical support from PEPFAR, USAID Global Health Supply Chain - Procurement and Supply Management program (GHSCPSM), Clinton Health Access Initiative (CHAI) and AIDS Healthcare Foundation (AHF) and had the following objectives: conduct on the spot assessment of uptake of new products (tenofovir/lamivudine/dolutegravir and Lopinavir/ritonavir $40 / 10 \mathrm{mg}$ ); ascertain reasons for the delay in transitioning of existing patients on previously preferred regimen tenofovir/lamivudine/efavirenz (TLE) and lamivudine/nevirapine/zidovudine (LNZ) to the new preferred regimen (TLD); strengthen quality of care at the antiretroviral treatment sites; identify laboratory related issues affecting quality of care and transition to TLD; mentor health care workers on documentation and reporting; and sensitize health care workers on pharmacovigilance for new products. This article highlights the processes of the Integrated national monitoring and supportive visit as conceived and introduced by NASCP and the observations and outcome from activity undertaken in 154 health facilities across 21 states of the federation (Abia, Adamawa, Akwa Ibom, Anambra, Bauchi, Benue, Cross River, Enugu, Gombe, Jigawa, Kaduna, Kano, Katsina, Zamfara, Lagos, Ogun, Nasarawa, Osun, Oyo, Rivers and Taraba).

\section{Methodology}

\section{Framework Development}

A team of technical staff from GHSC-PSM, CHAI and FHI360/Global Fund was constituted by NASCP to develop a framework that will guide the implementation of the iMSV. The team in collaboration with National AIDS and STIs Control Program (NASCP) and National Agency for the Control of AIDS (NACA) developed the National iMSV guideline, reporting templates (word and excel version), facility assessment checklist with a section on quality improvement plan, participants list and other supporting documents. Two meetings were held with all stakeholders in HIV programme to review and finalize the iMSV plan and other documents and to agree on funding for the activity. The guideline, checklist and other documents were adopted by the national HIV programme for implementation following the review. The checklist is sub-divided into different sections: Antiretroviral treatment (ART); Pharmacy; Laboratory; Regimen optimization and Records; and covers topics on (1) human resource, (2) service delivery \& adherence to standards, (3) pharmaceutical care service, (4) commodity Management, (5) documentation \& data Management and (6) adult and pediatric patients' optimization. The checklist also contains a scoring system wherein different indicators were measured to rank health facilities using a 3-point Likert scale, anchored by 'fully compliant (2)', 'partially compliant (1)' and 'noncompliant (0)'.

\section{Sites Selection}

Only health facilities providing comprehensive ART services were selected and prioritization was based on the following criteria: sites with population of patients on 
antiretroviral therapy (ART) above $\geq 1000$; sites without logistics Management information report (LMIS) in the last three cycles (July/August 2018, Sept/Oct 2018 and Nov/Dec 2018); sites with zero consumption of dolutegravir 50mg (DTG) and TLD from November/December 2018 LMIS data. Special consideration was given to states with highest HIV burden based on the report of Nigeria AIDS and Impact Indicator Survey (NAIIS). In total, 196 health facilities were selected after a pre-adjustment of the sample states.

Targeted population: Health workers directly involved in the management of HIV/AIDS programme in selected health facilities across the 21 states of the federation formed the population. They were drawn from various cadres ranging from Doctors, Pharmacists, Laboratory Scientist, and Medical Record Officers.

Data collection and other activities: Notification letter about the iMSV was sent by NASCP to the leadership of the Ministries of Health of the states involved in the exercise two weeks prior to the visits. The States Ministries of Health and the implementing partners supporting the selected health facilities similarly notified the health facilities one week before the exercise.

2-day orientation meetings on the iMSV were organized by NASCP for the team that supported the activity from the national level. The national team likewise held 2-hours abridged orientation meeting with the state team on the first day of the exercise before setting out for the site visit. The orientation aimed to strengthen the capacity of the participants to conduct iMSV to the health facilities to improve provision of health care services, build the knowledge, skills and attitude of the participants and so enable them to improve their iMSV practices and consequently make positive impacts on health worker and facility performance [1].

Before the iMSV, the State AIDS Programme Coordinator (SAPC) and the State Logistics Management Coordination Unit (LMCU) Coordinator of each States Ministries of Health prepared a matrix listing the dates the various health facilities will be visited, routes, vehicles for each trip, and members of the team.

The iMSV involved visits to selected health facilities by a mixed team of Government, funding agencies and nongovernmental organization (NGO) technical staff from national and state level from 8-12 th April 2019, to collect information on specific key \& quality issues as per structured checklists. The activities comprised on-site documentations, physical inventory, on-the-job training, demonstration and correction of wrong practices that can be address immediately, and sensitization of staff members on guidelines and correct processes. A quality improvement plan was prepared at each facility to address specific problems, while debriefing sessions with leadership of the health facilities were held following each site visit to discuss key findings, summarize field notes and observations. On the final day of the activity, each state team debriefed the leadership of states ministries of health providing general feedback, and suggestions for improvement. A total of 42 participants from the national level and 105 from state level participated in the iMSV to 154 health facilities across 21 states of the federation. There were 20 team overall and each team visited a maximum of 2 health facilities per day, while the visit lasted for an average of 3.5 hours in each health facility and took place in the ART, Pharmacy, Laboratory and Medical record.

The same process is to be repeated on biannual basis, to show improvement by time/intervention and existing gaps. Loevinsohn et al also found correlation between frequency of supervisory visits and improvements, suggesting that increasing the frequency of supportive visits helps only if the activities that occur during visits are productive and directly related to improving health worker performance [9].

Data analysis: Following data collection, editing and sorting of the checklists to determine the level of completeness, the information and data were entered into a data entry template. Data entry and analysis were performed using MS Excel. Descriptive data was presented in summary tables while numerical data was presented in graphs. The outcome was shared with states ministries of health implementing partners and health facilities for corrective actions.

\section{Results}

Of the 196 health facilities targeted for the iMSV, 154 were covered, giving a coverage rate of $78.6 \%$. Findings from the facility assessments were analyzed across all sections of the iMSV checklist-ART, Pharmacy, Laboratory and Records and presented in Figures 1-4. The figures highlight the weighted average (in \%) of the scores obtained by facilities on each question on the checklist. 


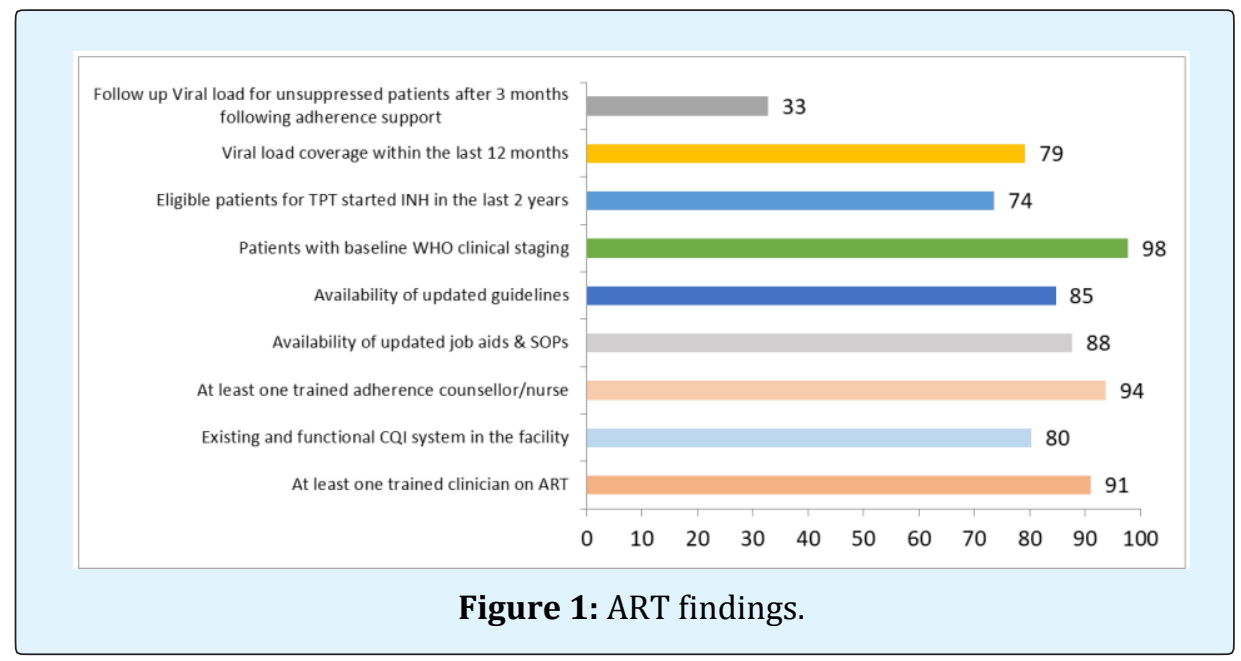

Most facilities had at least 1 adherence counsellor and 1 trained physician on ART and baseline WHO clinical staging is done for most patients before commencement on ART. Follow-up viral load testing for unsuppressed patients after adherence counselling is not done for most patients. Current national treatment guideline and required job aids and SOPs were generally available in most of the facilities. Viral load (VL) and Tuberculosis Preventive Therapy (TPT) coverage in most facilities is low and needs to improve. Greater number of the facilities had an existing and functional continuous quality improvement (CQI) system in place.

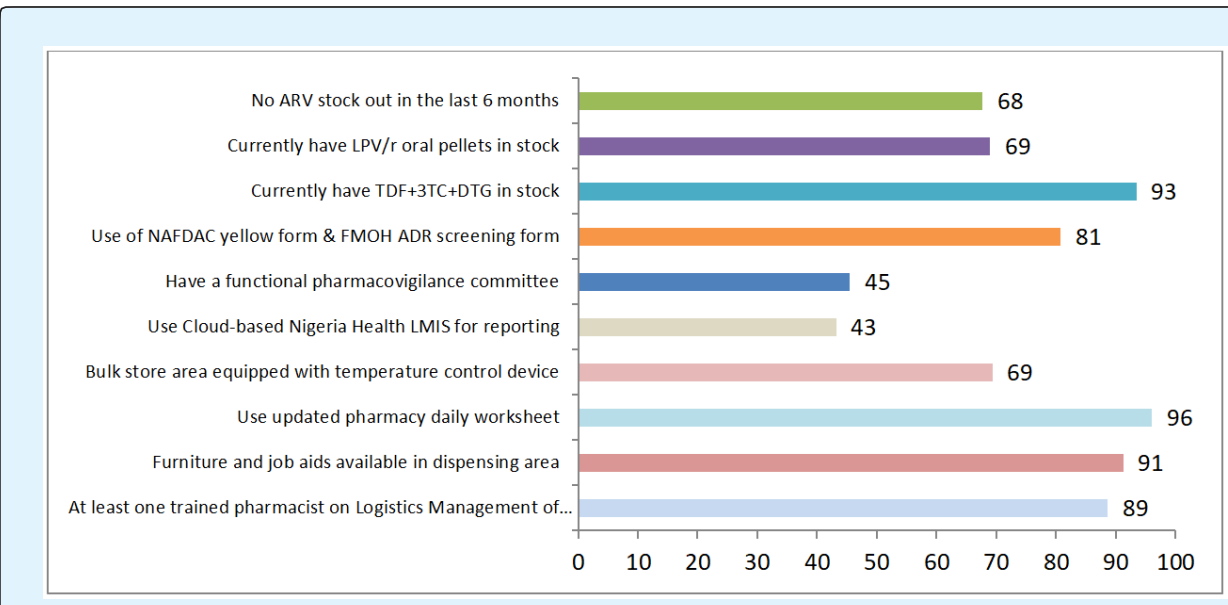

Figure 2: Pharmacy Findings.

Majority of the facilities had at least 1 Pharmacist trained on LMHC and TLD stock was generally available to support transition to the new regimen. However, only $69 \%$ of the facilities had stock of Lopinavir/ritonavir $40 / 10 \mathrm{mg}$. On the other hand, most pharmacovigilance committees were not functional, and majority of the facilities were not submitting LMIS through the cloudbased Nigeria Health LMIS. A good number of the facilities had their dispensing area equip with furniture and are using updated pharmacy worksheet for daily documentation. Greater number of the facilities are using National Agency for Food and Drug Administration and Control (NAFDAC) yellow form and Federal Ministry of Health (FMOH) adverse drug reaction (ADR) for ADR reporting and screening respectively. Incident of product stock out was reported in $32 \%$ of the facilities in the last 6 months while $69 \%$ of the facilities are maintaining good storage practices as they have their bulk store area equip with temperature control devise. 


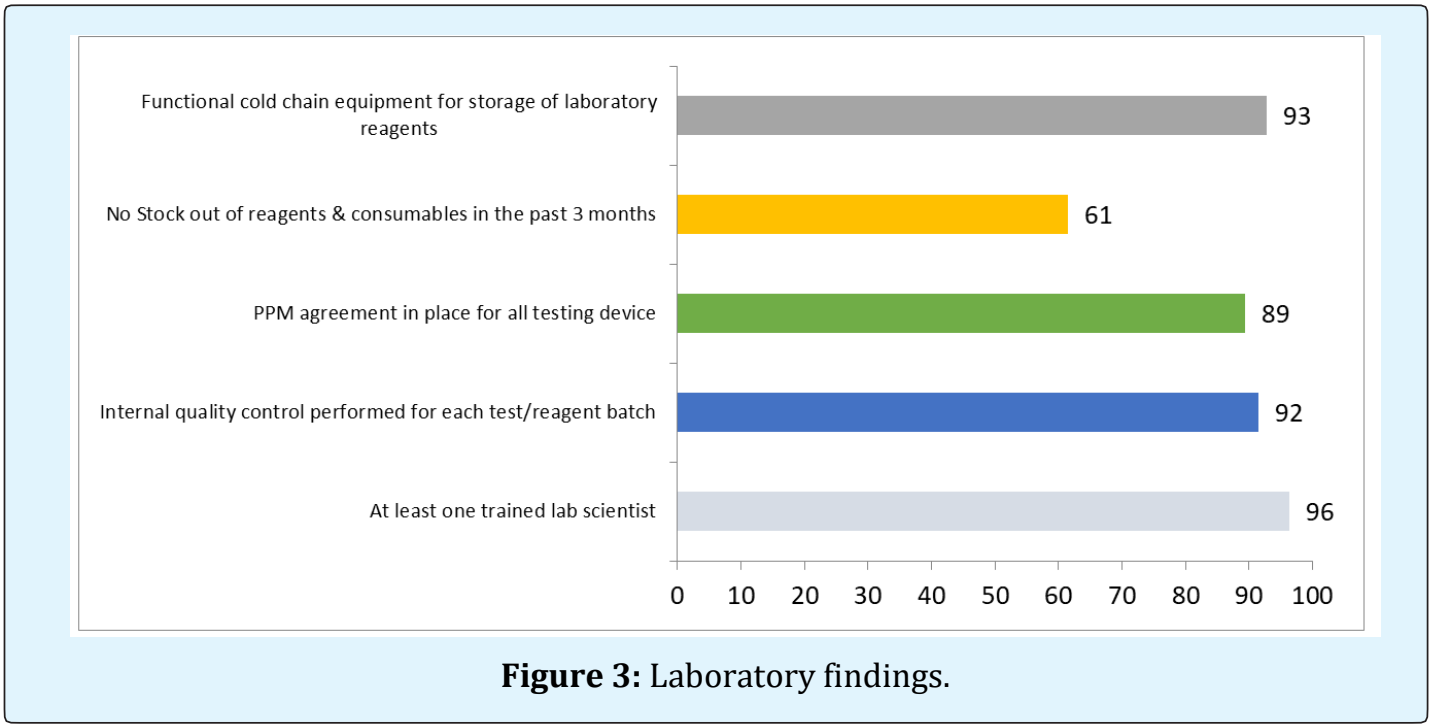

There was at least 1 trained lab scientist in most facilities. Incident of stock out of reagents and consumables was reported in $38 \%$ of the facilities in the last 3 months. 92\% perform internal quality control on each test/reagent batch before use. 89\% had plan preventive maintenance agreement is in place for the laboratory equipment while $93 \%$ had functional cold chain equipment for storage of laboratory reagents.

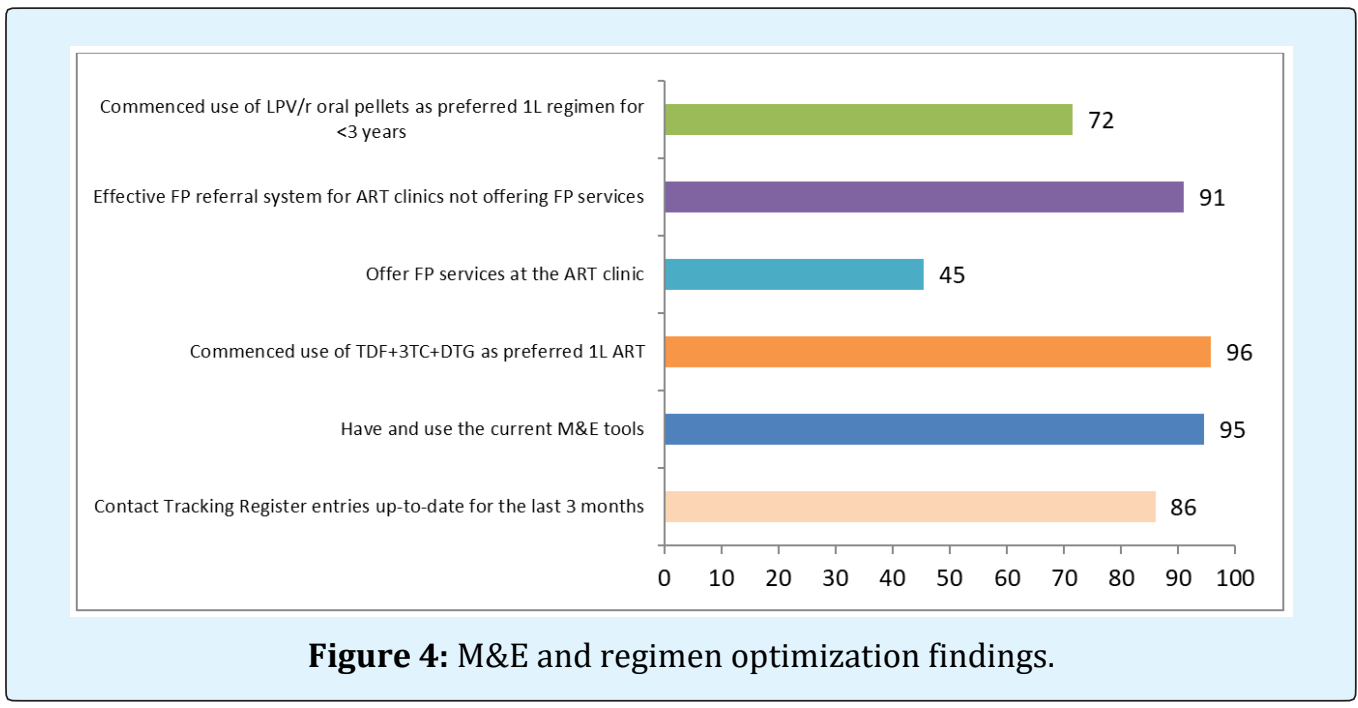

Current National M\&E tools are generally available and in use in most facilities. The use of TLD and LPV/r as preferred first line in adults and pediatric has commenced in most facilities. There is an effective referral system for family planning in ART clinic in most facilities, however only $45 \%$ of the facilities offers family planning services. Entries in contact tracking register were regularly updated in most of the facilities.

\section{Conclusion}

A key note behind success of the iMSV is that it was implemented by multiple stakeholders, including funders, implementing partners, Government of Nigeria staff and health care workers themselves, thereby addressing acceptance and ensuring ownership. It is evident from the findings that improvement in new products uptake and quality of care can be achieved by close monitoring, 
support and re-strengthening of the health care workers at the service delivery points. The iMSV achieved some notable successes, foremost were in promoting best practices for VL and TPT optimization, encouraging aggressive transition to new regimes and improving data quality. Optimization of viral load testing and TB preventive therapy (TPT) across the programme, advocacy for increase in number of qualified personnel at the ART clinics of the facilities for better service delivery, reactivation of continuous quality improvement, Regimen switch and Pharmacovigilance committees across facilities, capacity building and support for facility staff on reporting via the cloud-based Nigeria Health LMIS are key issues that needs to be followed up with the implementing partners and health facilities to improve quality of the programme.

Although finding revealed that most of the facilities had commenced the use of TLD and Lopinavir/ritonavir as the new preferred first line for adult and pediatric patients respectively, qualitative data showed a slow pace of transition of eligible existing patients to TLD especially women of reproductive age. But, there is insufficient quantitative data to determine the transition rate against set targets. The following will be required for effective implementation of future iMSV: adequate notification time for the States Ministries of Health and health facilities to ensure active participation; early communication of team compositions and schedule with all concerned stakeholders for adequate planning; participation of all implementing partners to facilitate access to their supported facilities. In nutshell, iMSV can serve as a useful tool for the programme, since it serves to collect and analyze information, and generate feedback for corrective action and monitor improvement during subsequent visits.

\section{Disclaimer}

The findings and conclusions in this paper are those of the authors and do not necessarily represent the official position of the USAID or GHSC-PSM.

\section{Competing Interests}

The authors of this manuscript declare that they have no competing interests.

\section{Acknowledgement}

The authors would like to acknowledge all the stakeholders that participated in the National Integrated
Monitoring and Supportive Visits especially CHAI and NASCP.

\section{References}

1. Madede T, Sidat M, McAuliffe E, Patricio SR, Uduma O, et al. (2017) The impact of a supportive supervision intervention on health workers in Niassa, Mozambique: a cluster-controlled trial. Human Resources for Health 15(1): 58.

2. Vijaya KM, Ram PM, Bala KC (2015) Supportive Supervision as a Tool to Improve and Monitor Progress the Quality of Immunization Services in India. International Journal of Pharmaceutical and Medical Research 3(2).

3. Marquez L, Kean L (2002) Making Supervision Supportive and Sustainable: New Approaches to Old Problems, Maximizing Access and Quality Initiative, MAQ Paper No. 4, USAID, Washington DC.

4. Ali BQ, Naqvi H, Zahida S, shtiaq A (2016) Role of Monitoring and Supervision to Improve Health Service Delivery in Basic Health Units of Punjab, Pakistan.

5. Hernandez AR, Hurtig AK, Dahlblom K, San SM (2014) More than a checklist: a realist evaluation of supervision of mid-level health workers in rural Guatemala. BMC Health Services Research, pp: 14112.

6. Clements CJ, Streefland PH, Malau C (2007) Supervision in primary health care--can it be carried out effectively in developing countries? Current Drug Safety 2(1): 19-23.

7. PATH (2003) Children's Vaccine Program at PATH. Guidelines for Implementing Supportive Supervision: A step-by-step guide with tools to support immunization.

8. Kwik-Skwiz \#15 (1998) Supporting Staff through Effective Supervision: How to Assess, Plan and Implement More Effective Clinic Supervision. Health Systems Trust.

9. Loevinsohn BP, Guerrero ET, Gregorio SP (1995) Improving Primary Health Care through Systematic Supervision: A Controlled Field Trial. Health Policy and Planning 10(2): 144-153. 
